\title{
Comparison Between Internal Jugular and Upper Arm Peripheral Veins Approach in Insertion of Totally Implantable Central Venous Ports
}

\author{
Amr Nabil, MD, MSc; ${ }^{1}$ Mohammed Zaki, MD, MSc, MRCS; ${ }^{1}$ Abdallah Gamal, MSc $^{2}$ \\ ${ }^{1}$ Department of Vascular Surgery, Ain Shams University, Egypt \\ 2Department of Vascular Surgery, Gamal Abd-Elnaser Hospital, Alexandria, Egypt
}

\begin{abstract}
Objectives: To compare internal jugular vein approach and Upper arm approach through basilic or cephalic veins in insertion of total implantable central venous port regarding early post-operative complications, patency rate, compliance and patient quality of life.
\end{abstract}

Patients and methods: We identified 50 patients who underwent totally implantable venous access ports (TIVAP) implantation in the arm (25 patients) or chest (25 patients) between November 2015 and November 2017. Implantation via an upper arm (cephalic or basilic) occurred through venous cut down, the internal jugular vein approach was performed using percutaneous technique. ${ }^{1}$ All approaches were Under fluoroscopic guidance. Early post-operative Complications were evaluated. During follow up, self-compliance and quality of life were assessed as well.

Results: Technical success was $100 \%$. Procedure-related arterial injury occurred in 3 patients in central approach only. Post-operative hematoma, stitch inflammation and seroma were observed. Late complications including catheter infection, occlusion, pinch off syndrome, skin dehiscence, thrombophlebitis of the vein and extravasation in both technique was documented, and quality of life was assessed during follow up.

Conclusions: Totally implantable venous access ports (TIVAP) can be implanted with high technical success rates and are associated with low rates of complications. Upper arm implantation may benefit clinicians and patients with respect to safety and comfort.

Key words: Central venous catheters, peripheral portacath, chemotherapy.

\begin{abstract}
Introduction
The number of cancer patients has been increasing worldwide due to progressive society ageing. Rapid developments in outpatient cancer chemotherapy have exponentially increased the need for implantable central venous (CV) ports. ${ }^{2}$ Totally implantable venous access ports (TIVAP) are widely used and allow for administration of chemotherapy and artificial nutrition as well as blood sampling. These devices have been evaluated extensively in various locations, e.g. the chest, upper arm and forearm, generally showing excellent results as to technical success and low rates of complications with the reservoir positioned in the $\mathrm{arm}^{3}$ The potential benefits that justify a more detailed study of this technique include reducing the risk of intraoperative complications such as arterial injury, pneumothorax or hemothorax, lack of interference in breast imaging, easier access to puncture, better cosmetic results and better quality of life. ${ }^{4}$
\end{abstract}

\section{Methods and materials}

In this retrospective study, we identified 50 patients who underwent percutaneous TIVAP implantation between November 2015 and November 2017 in Gamal Abd-Elnaser Hospital, Alexandria and Ain Shams University Hospitals and were suffering from different neoplastic diseases requiring chemotherapy. Patients' demographic and baseline characteristics are highlighted in (Tables 1-3). Indication for TIVAP insertion was to administer chemotherapy. All patients were examined and treated as part of routine care, and all patients signed an informed consent for the procedure. Institutional review board approval was not required. 25 patients were men and 25 were women. 24 Patients presented with cancer colon (48\%) (11 central and 14 peripheral), 4 patients with cancer stomach $(8 \%)$ ( 2 central and 2 peripheral), 2 with bone cancer (4\%) (one central and one peripheral), 4 with cancer esophagus (8\%) (3 central and one peripheral), 10 with cancer breast (20\%) (5 central and 5 peripheral) and 6 with other malignances (12\%). 25 patients had central approach while the other 25 patients had peripheral approach, (18 of them were basilic and the other 7 patients were cephalic). 
Table 1: Patients' demographic and baseline characteristics

\begin{tabular}{llcc}
\hline & Variable & Number & Percent \% \\
\hline \multirow{2}{*}{ Gender } & Male & 25 & $50 \%$ \\
& Female & 25 & $50 \%$ \\
\multirow{3}{*}{ Malignancy } & Cancer colon & 24 & $48 \%$ \\
& Cancer stomach & 4 & $8 \%$ \\
& Cancer bone & 2 & $4 \%$ \\
& Others & 6 & $12 \%$ \\
Access site & Cancer Esophagus & 4 & $8 \%$ \\
& Cancer Breast & 10 & $20 \%$ \\
& Central & 25 & $50 \%$ \\
\end{tabular}

Table 2

\begin{tabular}{lcccc}
\hline & Minimum & Maximum & Mean & SD \\
\hline Age & 43 & 67 & 55.32 & 6.103 \\
Hemoglobin & 9 & 12 & 10.56 & 0.686 \\
PLT & 153000 & 350000 & 242360 & 43957 \\
INR & 1 & 1.2 & 1.02 & 0.043 \\
Prothrombin Activity & 90 & 100 & 99.38 & 1.665 \\
Cephalic vein diameter & 1.6 & 3 & 2.18 & 0.377 \\
Basilic vein diameter & 1.8 & 3.5 & 2.88 & 0.332 \\
\hline
\end{tabular}

Table 3

\begin{tabular}{lcc}
\hline Malignancy & Central & Peripheral \\
\hline Cancer colon & 11 & 13 \\
Cancer Esophagus & 2 & 2 \\
Cancer bone & 1 & 1 \\
Cancer Breast & 5 & 5 \\
Cancer stomach & 3 & 1 \\
Others & 3 & 3 \\
\hline
\end{tabular}

\section{Description of upper arm central venous port implantation}

The access route of choice was the right arm as shown in (Figure 1), the preparation for surgery included shaving the catheter implantation site when necessary, and disinfection of the entire limb with Povidone Iodine.

To reduce the difficulty in obtaining the vein by cut down technique, intraoperative duplex was used to determine the site of cut down on the basilic or the cephalic veins.
In the operation room, the patient should lie down in the supine position, allowing the upper limb to be abducted rotated outwards in basilic and inwards in cephalic approach, forearm to supinate in basilic or pronate in cephalic approach, and medial side of the arm to be upward for better demonstration of the basilic vein. The elbow should not be bent, and the forearm should not be pronated in basilic approach.

\section{Central venous catheter placement and port implantation}

The point for a skin incision was 3-4 fingers breadth from medial or lateral epicondyle of the arm. 
Local anesthetic was applied to areas about $2 \mathrm{~cm}$ right and left from the point of skin incision and to areas $2 \mathrm{~cm}$ peripheral from these to establish a subcutaneous pocket. Subsequently, a scalpel was used to make a skin incision extending $2 \mathrm{~cm}$ to the right to $2 \mathrm{~cm}$ to the left of the incision point. This incision was later used as the entrance for making a subcutaneous pocket with a forceps. The connective tissues between the skin and the basilic vein was then removed and the basilic vein was identified and accessed through a peripheral 22 gauge cannula.

A $0.014 \mathrm{~mm}$ guidewire was inserted through the lumen of the peripheral cannula placed in the vein and negotiated proximally until SVC is reached under $\mathrm{X}$-ray fluoroscopic guidance. If there was abnormal resistance during wire passage, appropriate use of a contrast injection was performed to confirm a run-through of the vessel and presence of stenosis or occlusion. After introducing the guidewire, the peripheral cannula was withdrawn and replaced by the sheath, followed by advancement of the catheter over the wire through the sheath.

The catheter was appropriately positioned in the SVC. The optimal CV catheter tip location was about $2 \mathrm{~cm}$ passed centrally from the SVC confluence, as recognized by fluoroscopy. The sheath was peeled off.

A subcutaneous pocket for a port was made by blunt dissection using forceps (Figure 2). The port and catheter were then connected according to the manufacturer's manual. Fixing the port to connective tissue through the suture hole was optional with our upper arm method according to the pocket size. Finally, the skin was sutured appropriately while avoiding pricking the catheter.

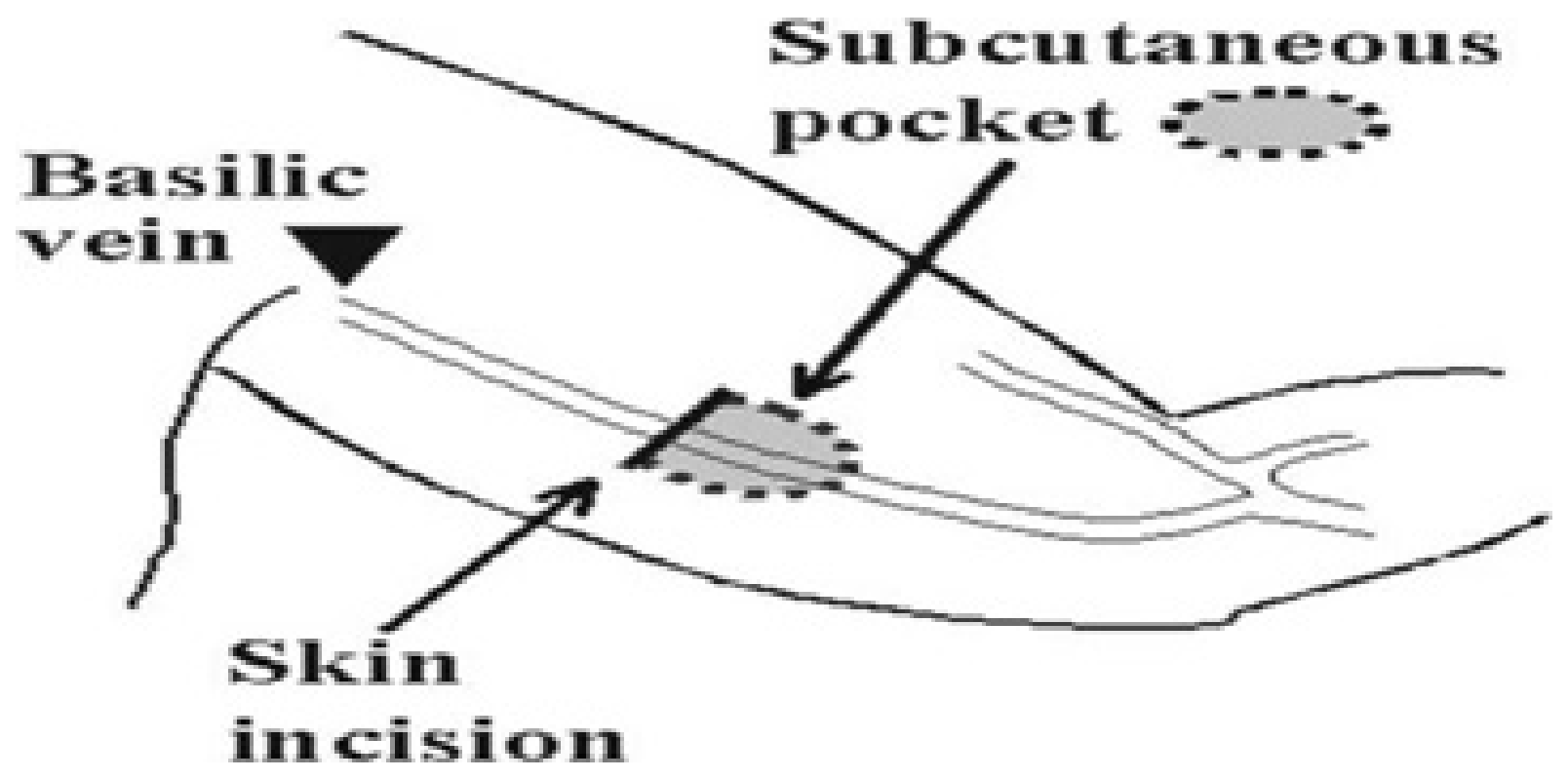

Fig 1: Description of site of peripheral basilic insertion.

\section{For pectoral placement}

Following local anesthesia, the internal jugular vein was accessed using the percutaneous technique with micro puncture needle. Subsequently, the wire was introduced and confirmed that it is in the correct position under fluoroscopic guidance.

Following preparation of the port pocket in the chest, a tunneling trocar was used to cross the distance between the pocket and the initial puncture site subcutaneously.
The peel-away sheath was placed in the internal jugular vein. The catheter tip was inserted via the sheath under fluoroscopic guidance and placed centrally with the tip aiming at the vertebral body below the SVC confluence.

After tunneling the distance between the initial vascular access site and the pocket, the catheter was transected to adequate length and connected to the port chamber. Correct and central placement of the catheter tip as well as the loop-free run of the catheter in the tunneled area was documented by 
fluoroscopy.

The port was fixed to the pectoral muscle fascia by proline suture $4 \backslash 0$. The pocket was closed with one layer of suture, as the vascular access site was closed with a single cutaneous stitch.

At the end of all procedures, all TIVAP were accessed with a non-coring puncture needle. Before needle removal, the catheter was flushed with heparinized saline solution. Following pectoral implantation, pneumothorax was ruled out by chest X-ray after expiration.

Intraoperative data, such as operating time, type of anesthesia, access route changes and intraoperative complications were recorded for further evaluation.

Patients were instructed to keep applying sterile occlusive dressings for 3 days after the procedure. In case of need for immediate use of the device, the first puncture was performed in the surgical room.

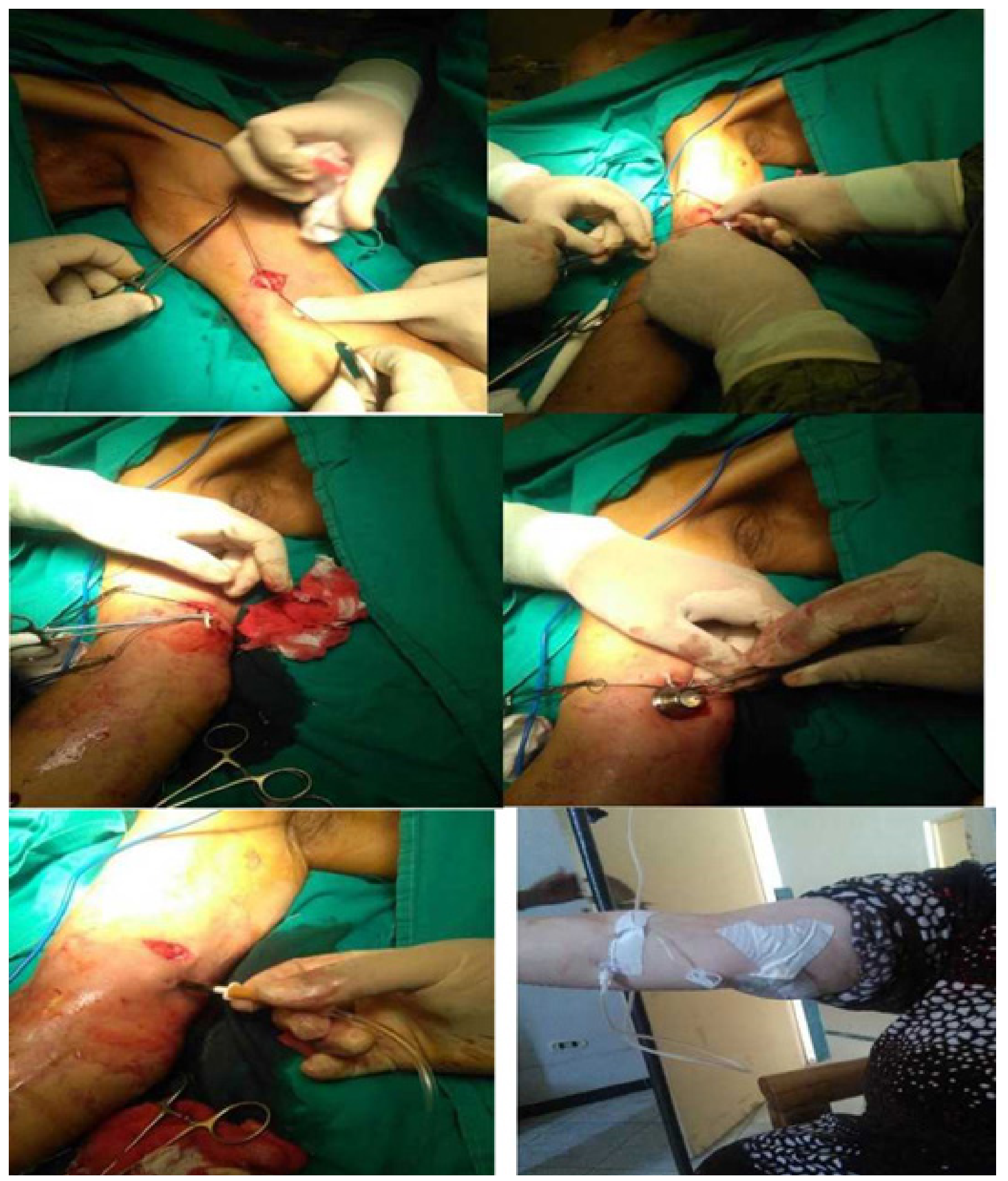

Fig 2: Steps of peripheral port insertion A: Identifying basilic vein B: Introduction of tube over the wire $C$ and D: Connection of the tube and port fixation subcutaneously. E: Final test. F: Patients has chemotherapy through the port. 


\section{Follow up}

Patients included in the sample were clinically evaluated at 10 and 30 days, 3 months and 6 months after the procedure and at the end of chemotherapy or at any other time of the study in case of any catheter-related intercurrent events.

Additional tests such as X-ray or Doppler ultrasound were requested only if the patient complained of symptoms related to the catheter (e.g., dysfunction, edema or changes related to the surgical wound).

The primary outcomes assessed were the early postoperative complications, defined as events occurring within 30 days after implantation and late complications which occurred after that.

A patient satisfaction questionnaire was fulfilled in the second evaluation after 30 days of implantation.
From the patients' perspective, this questionnaire analyzed data involving recognition of the need for the device, aspects of comfort; anxieties generated by the use of the device; interference in daily activities; aesthetics and overall satisfaction based on the recommendation grade indicated by the patient.

The patients were asked whether they agreed or disagreed with statements relating to the different aspects of satisfaction analyzed and the results of the questionnaire were compared.

\section{Results}

In preprocedural period 18 patient $(36 \%)$ in the group of central approach required $\mathrm{O}_{2}$ mask in Trendelenburg positioning while no one in peripheral approach required $\mathrm{O} 2$ mask or Trendelenburg position ( $p$ value was $<0.001$ ).

Table 4: Difficulties during operation requiring $\mathrm{O}_{2}$ mask

\begin{tabular}{ccccc}
\hline & & \multicolumn{3}{c}{$\begin{array}{c}\text { Difficulties during operation requiring } \mathbf{O}_{\mathbf{2}} \\
\text { mask }\end{array}$} \\
\cline { 3 - 4 } & & Yes & No & \\
\cline { 3 - 4 } & & $18(72 \%)$ & $7(28 \%)$ & $<0.001^{* *}$ \\
Access site & Central (25pt) & $4(16 \%)$ & $21(84 \%)$ & \\
Total & Peripheral (25pt) & $22(44 \%)$ & $28(56 \%)$ & \\
\hline
\end{tabular}

During the operation, technical success was $100 \%$. There were no intraoperative complications in 47 patients $(94 \%) 25(100 \%)$ of the peripheral approach and 22 (88\%) of central approach. 3pts $(12 \%)$ in central approach had accidental arterial puncture during cannulation of jugular vein by the needle which was controlled by compression followed by correct cannulation of the jugular vein. None of the patients had pneumothorax or hemothorax ( $p$ value 0.037 ).

Table 5: Intraoperative complications

\begin{tabular}{ccccccc}
\hline & & \multicolumn{3}{c}{ Intraoperative complications } \\
\cline { 3 - 7 } & & None & $\begin{array}{c}\text { Arterial } \\
\text { injury }\end{array}$ & Pneumothorax & Hemothorax & P-value \\
\hline Access & $\begin{array}{c}\text { Central } \\
(25 \text { patients })\end{array}$ & $22(88 \%)$ & $3(12 \%)$ & 0 & 0 & \\
Site & $\begin{array}{c}\text { Peripheral } \\
(25 \text { patients })\end{array}$ & $25(100 \%)$ & 0 & 0 & 0 & 0.037 \\
Total & 50 patients & $47(94 \%)$ & $3(6 \%)$ & 0 & 0 & \\
\hline
\end{tabular}

During the post-operative period a total number of 37 patients $(74 \%)$ (18 patients in central $(72 \%)$ and 19 patients (76\%) in peripheral approach) had no post-operative complications, 4 patients $(8 \%)$ (3 patients (12\%) in central and 1 patient (4\%) in peripheral approach) had postoperative hematomas which resolved by conservative treatment. Seven patients (14\%) $(4-16 \%$ in central and $3-12 \%$ in peripheral) had wound infection in the form of suture line inflammation and were managed with conservative treatment through oral antibiotics. One patient in the peripheral approach $(4 \%)$ had wound hematoma which was superimposed by infection and required bedside evacuation and antibiotics followed by antibiotic therapy. The port was not removed and after 3 weeks of treatment, the patient initiated the chemotherapy through the port with no further complications. One patient in the peripheral approach (4\%) had wound seroma which was treated by slight compression on the wound by dressing and crepe bandage for two weeks and oral antibiotics. The port was not removed and after 5 weeks of treatment the patient initiated the chemotherapy through the port with no further complication. 


\begin{tabular}{|c|c|c|c|c|c|c|c|}
\hline & & \multicolumn{5}{|c|}{ Postoperative complications } & \multirow[b]{2}{*}{ P-value } \\
\hline & & None & Hematoma & Wound infection & $\begin{array}{l}\text { Hematoma \& wound } \\
\text { infection }\end{array}$ & Seroma & \\
\hline \multirow{2}{*}{ Access site } & Central (25 pt) & $18(72 \%)$ & $3(12 \%)$ & $4(16 \%)$ & 0 & 0 & \multirow{3}{*}{0.407} \\
\hline & Peripher al (25 pt) & $19(76 \%)$ & $1(4 \%)$ & $3(12 \%)$ & $1(4 \%)$ & $1(4 \%)$ & \\
\hline Total & 50 patients & $37(74 \%)$ & $4(8 \%)$ & $7(14 \%)$ & $1(2 \%)$ & $(2 \%)$ & \\
\hline
\end{tabular}

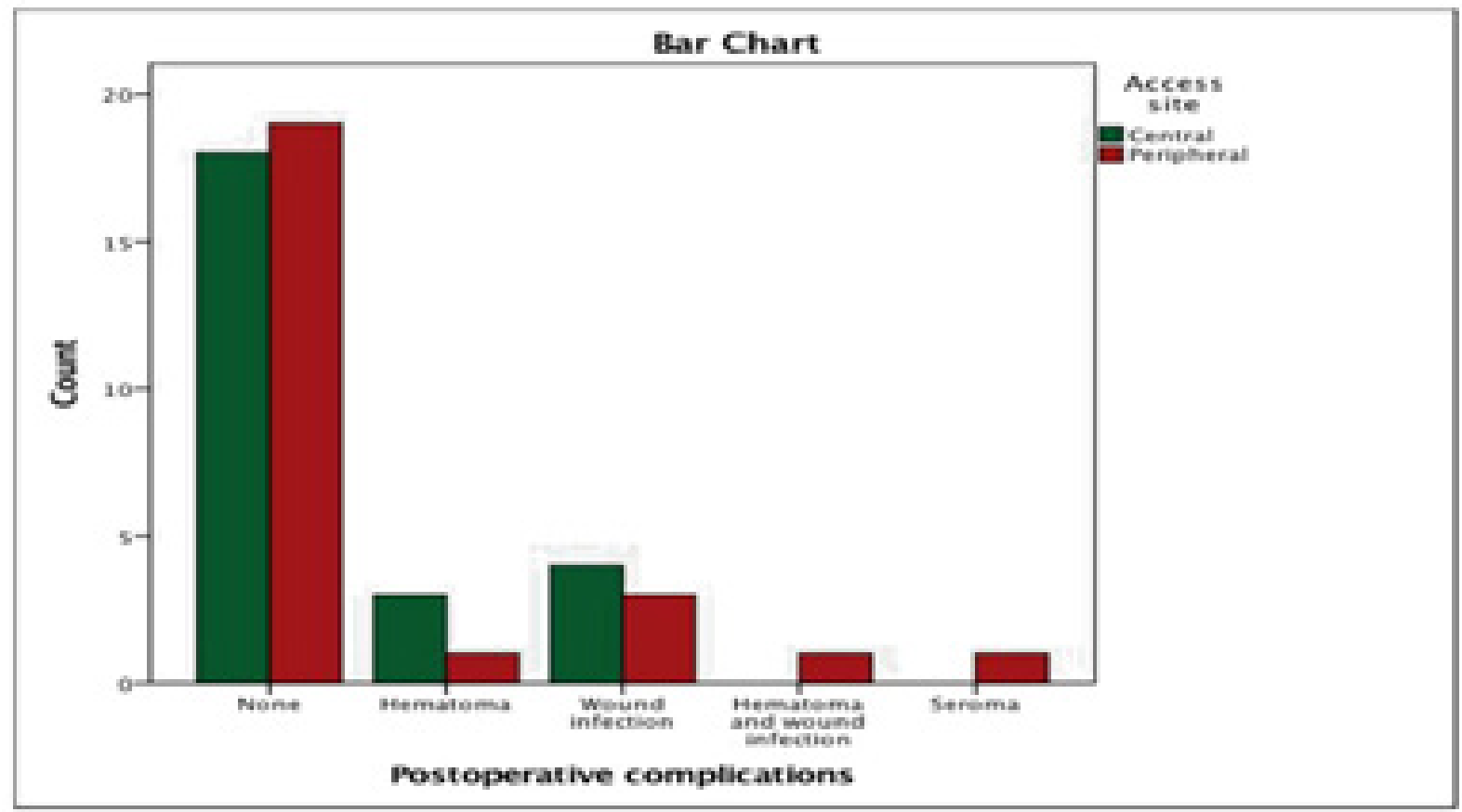

Fig 3: Post operative complications.

Regarding late complications a total number of 37 (74\%) patients $18(72 \%)$ central and 19 (76\%) peripheral) approach had no late complications, while 4 patients (8\%) $3(12 \%)$ in central and $1(4 \%)$ in peripheral) approach had catheter infection which ended by catheter removal in $2 \mathrm{pt}$ of central and $1 \mathrm{pt}$ of peripheral and conservative tt with antibiotics in one patients with central approach.

Thrombo-phlebitis of the vein occurred only in peripheral approach in 3pts (12\%) who were treated conservatively by anti-inflammatory medications. The port was not removed and the patients continued to have chemotherapy with central approach. Cath occlusion occurred only in 3pts (12\%) and the port was removed. Extravasation occurred in one patient $(4 \%)$ due to wrong needle placement by the nurse and passed conservatively. Wound dehiscence occurred in 2 pts ( $8 \%$ ) and passed conservatively with cessation of therapy through the port for 3 weeks. Also port trauma occurred in one patients $(4 \%)$ in central approach with $p$ value 0.014 .

Table 7: Late complications

\begin{tabular}{ccccccccc}
\hline \multicolumn{1}{c}{ Late complications } \\
\hline & None & $\begin{array}{c}\text { Cath } \\
\text { Infection }\end{array}$ & $\begin{array}{c}\text { Thrombo } \\
\text { phlebitis }\end{array}$ & Extravasation & Occlusion & $\begin{array}{c}\text { Wound } \\
\text { dehiscence }\end{array}$ & Trauma P-value \\
\hline \multirow{2}{*}{ Access site } & $\begin{array}{c}\text { Central } \\
\text { approach } \\
\text { Peripheral }\end{array}$ & $15(60 \%)$ & $3(12 \%)$ & 0 & $1(4 \%)$ & $3(12 \%)$ & $2(8 \%)$ & $1(4 \%)$ \\
Total & $21(84 \%)$ & $1(4 \%)$ & $3(12 \%)$ & 0 & 0 & 0 & 0 & $0.014 * *$ \\
& $\begin{array}{c}\text { approach } \\
\text { Topatients }\end{array}$ & $36(72 \%)$ & $4(8 \%)$ & $3(6 \%)$ & $1(2 \%)$ & $3(6 \%)$ & $2(4 \%)$ & $1(2 \%)$ \\
\hline
\end{tabular}




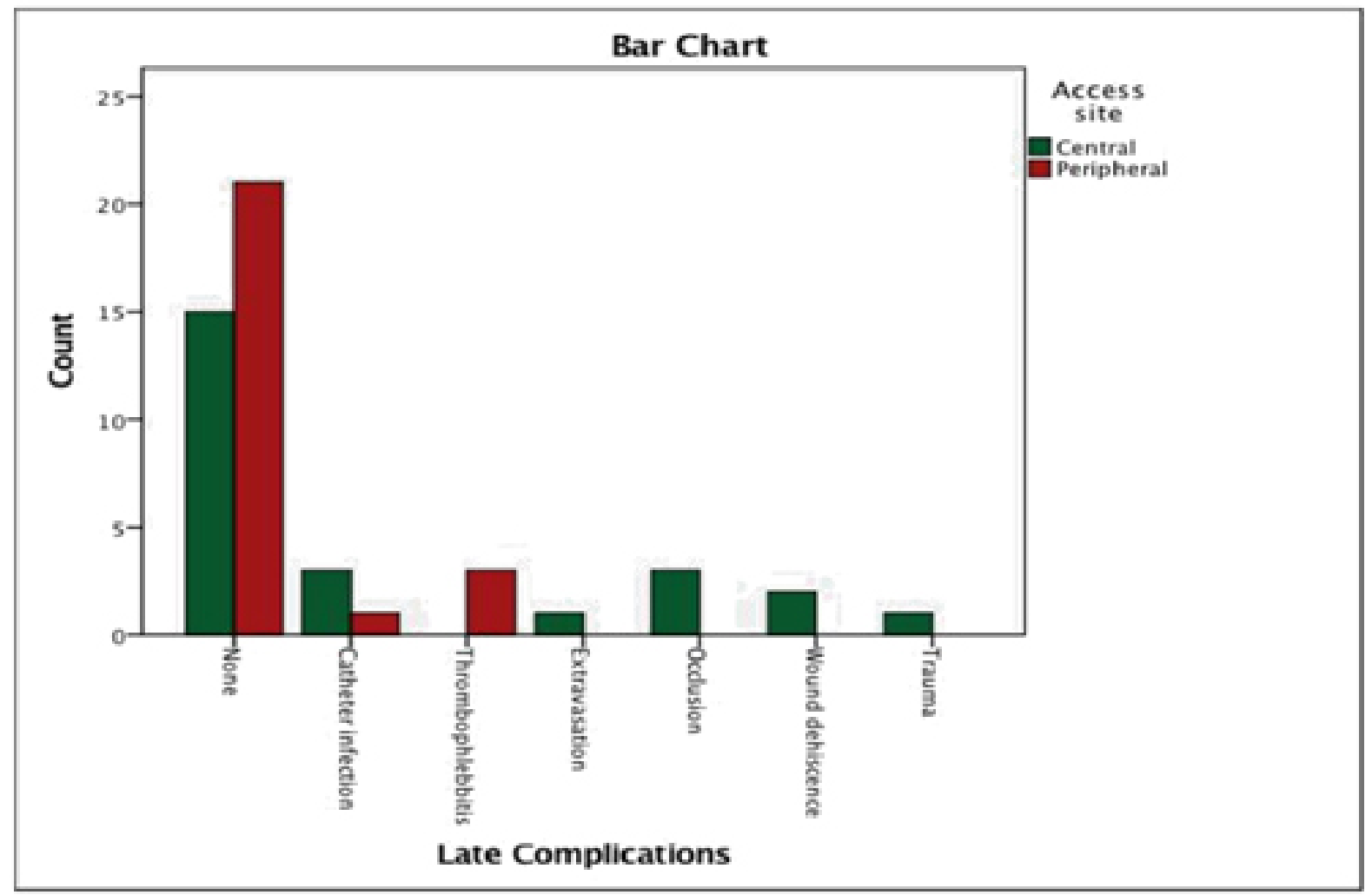

Fig 4: Late complications.

Cather removal occurred in total of 15 patients (30\%) 9 patients (18\%) ( 4 central and 5 peripheral) at the end of chemotherapy, $3(6 \%)$ (2 in central approach and one in peripheral approach) due to catheter infection and 3 in central approach due to catheter occlusion. Thirty five patients (70\%) chose to keep the port after the end of the chemotherapy because fearing of relapse of malignancy with $p$ value 0.18 .

Table 8: Catheter fate

\begin{tabular}{lccccc}
\hline & \multicolumn{3}{c}{ Catheter Removal } & & \\
\hline & $\begin{array}{c}\text { Cath } \\
\text { occlusion }\end{array}$ & $\begin{array}{c}\text { End of } \\
\text { therapy }\end{array}$ & Infection & $\begin{array}{c}\text { Patient } \\
\text { requested to } \\
\text { keep the port }\end{array}$ & P-value \\
\hline Access site & $\begin{array}{c}\text { Central } \\
(25 p t) \\
\text { Peripheral } \\
(25 p t)\end{array}$ & $3(12 \%)$ & $4(16 \%)$ & $2(8 \%)$ & $16(64 \%)$ \\
Total & 50 patients & $3(6 \%)$ & $9(18 \%)$ & $3(6 \%)$ & $35(70 \%)$ \\
\hline
\end{tabular}

\section{Procedure and quality of life}

During follow up, patients were asked if the port caused any unpleasant feeling. One patient $(4 \%)$ in the central approach group said yes, 16 (64\%) said no and $8(32 \%)$ said sometimes, while in peripheral approach $16(64 \%)$ said no and $9(36 \%)$ said sometimes with $p$ value 0.486 . 
Table 9: Patient's satisfaction - complaints of unpleasant feeling

\begin{tabular}{cccccc}
\hline & & \multicolumn{2}{c}{ Port causes unpleasant feeling } & P-value \\
\cline { 3 - 4 } & & Yes & No & Sometime & \\
\hline \multirow{2}{*}{ Access site } & Central (25pt) & $1(4 \%)$ & $16(64 \%)$ & $8(32 \%)$ & $0.486 * *$ \\
total & Peripheral (25pt) & 0 & $16(64 \%)$ & $9(36 \%)$ & $17(34 \%)$ \\
\hline
\end{tabular}

When patients were asked if the port caused pain when they had chemotherapy, a total number of 46 ( $92 \%$ ) said no (24-96\% central, $22-88 \%$ peripheral) and a total of $4(8 \%)$ patients (one- $4 \%$ central, $3-12 \%)$ said sometimes with $p$ value 0.287 .

Table 10: Port causing pain

\begin{tabular}{llccc}
\hline & No & \multicolumn{2}{c}{ Port causing pain } \\
\cline { 3 - 4 } & $\begin{array}{llc}\text { Central } 25 \mathrm{pt} \\
\text { peripheral }\end{array}$ & $24(96 \%)$ & Sometime & p-value \\
Access site & $25 \mathrm{pt}$ & $22(88 \%)$ & $3(12 \%)$ & $0.287^{* *}$ \\
total & $50 \mathrm{pt}$ & $46(92 \%)$ & $4(8 \%)$ & \\
\hline
\end{tabular}

Regarding patients' daily activities, all patients reported normal activities with regards to exercises or taking showers. All female patients stated they could wear bras normally except for one patient in the central approach in whom wearing a bra causes some discomfort $\mathrm{p}$ value was 0.183 .

Table 11: Patient's ability to take showers

\begin{tabular}{|c|c|c|c|}
\hline \multirow{8}{*}{ Access site } & \multicolumn{3}{|c|}{ Patient's ability to take showers } \\
\hline & & No & Yes \\
\hline & Central & 0 & $25(100 \%)$ \\
\hline & peripheral & 0 & $25(100 \%)$ \\
\hline & \multicolumn{3}{|c|}{ Patient's ability to exercise } \\
\hline & & No & Yes \\
\hline & Central & 0 & $25(100 \%)$ \\
\hline & peripheral & 0 & $25(100 \%)$ \\
\hline
\end{tabular}

Table 12: Females wearing Bras

\begin{tabular}{ccccc}
\hline & & \multicolumn{2}{c}{ Wearing Bras } & \multirow{2}{*}{ P-value } \\
\cline { 3 - 4 } & & Yes & No & \multirow{2}{*}{$0.183^{* *}$} \\
\cline { 2 - 4 } Access site & Central & $10(90.9 \%)$ & $1(9.1 \%)$ & 0 \\
\hline
\end{tabular}


A total number of 33 patients (66\%) (22-88\% in central and $11-44 \%$ patients in peripheral approach) said that during their daily activities they did not fear of port trauma or take extra care, while 8 patients
$(16 \%)$ ( $3-12 \%$ patients in central approach and $5-20 \%$ patients in peripheral approach) stated that they took extra precautions for fear of port trauma with $p$ value $<0.0001$.

Table 13: Fear of port trauma

\begin{tabular}{cccccc}
\hline & & \multicolumn{3}{c}{ Fear of port trauma } & P-value \\
\cline { 3 - 5 } & & Yes & No & Sometime & \\
\hline \multirow{3}{*}{ Access site } & Central 25 pt & $3(12 \%)$ & $22(88 \%)$ & 0 & $<0.0001^{* *}$ \\
& Peripheral 25 pt & $5(20 \%)$ & $11(44 \%)$ & $9(36 \%)$ & $9(18 \%)$ \\
\hline
\end{tabular}

When patients were asked about the site of port insertion during follow up in central approach 3 (12\%) patients prefered this site, 7 (28\%) prefered the peripheral approach and $15(60 \%)$ said that it had no difference while in peripheral approach 19 pt $(76 \%)$ prefered this site and 9 pt $(18 \%)$ prefered the central approach while $4 p t(16 \%)$ said that it had no difference with $p$ value $<0.0001$.

Table 14: Patient retrospective preference

Patient retrospective preference

$\begin{array}{cccc}\text { Preferred his } & \text { Preferred the other } & \text { No difference } & \text { P-value } \\ \text { designated site } & \text { site } & \text { Nolute }\end{array}$

\begin{tabular}{|c|c|c|c|c|c|}
\hline & Central (25pt) & $3(12 \%)$ & $7(28 \%)$ & $15(60 \%)$ & \\
\hline \multirow[t]{2}{*}{ Access site } & & & & & $<0.0001^{* *}$ \\
\hline & Peripheral (25pt) & $19(76 \%)$ & $2(8 \%)$ & $4(16 \%)$ & \\
\hline Total & 50 ptients & $22(44 \%)$ & $9(18 \%)$ & $19(38 \%)$ & \\
\hline
\end{tabular}

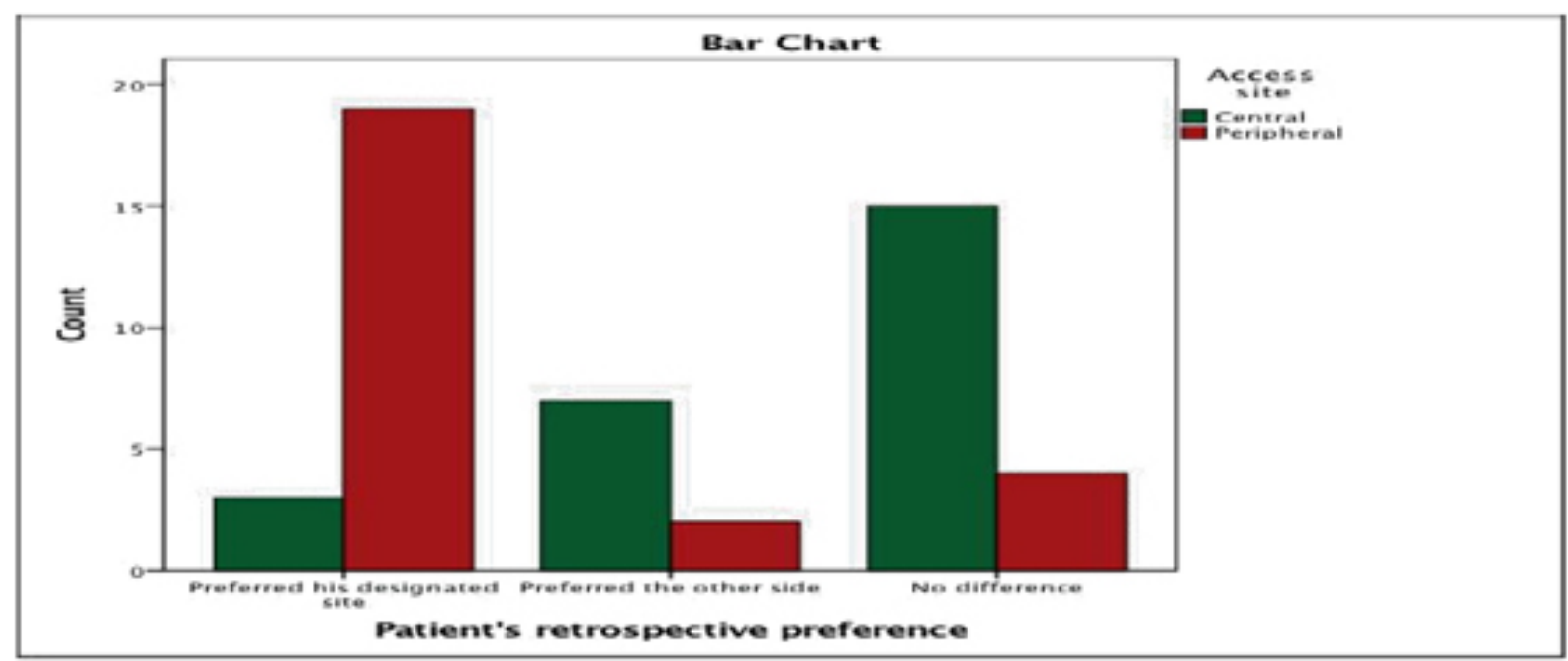

Fig 5: Patients retrospective preference. 
When the surgical team members were asked about the procedure, surgeons said that there were some difficulties in one patients in central approach and 3 patients in peripheral approach while no difficulties in 24 patients central and 22 in peripheral approach with $p$ value 0.287 .

Table 15: Difficulties to surgeon

\begin{tabular}{llccc}
\hline & & \multicolumn{2}{c}{ Difficulties to surgeon } & No \\
\cline { 3 - 4 } & & Yes & \multirow{2}{*}{ P-value } \\
\hline \multirow{2}{*}{ Access site } & Central & $1(4 \%)$ & $24(96 \%)$ & $0.287^{* *}$ \\
\multirow{2}{*}{ Total } & peripheral & $3(12.3 \%)$ & $22(88 \%)$ & \\
\hline
\end{tabular}

When the nurses who give patients chemotherapy were asked about their opinion about the feasibility of the new approach, they stated that they have difficulties in cannulation in the first 4 patients $(16 \%)$ and then had no difficulties dealing with the other $21(84 \%)$ patients in the peripheral approach, while they didn't have any difficulties with patients with central approach; and this indicates the importance of the learning curve in dealing with the new approach.

Table 16: Difficulties in usage with nursing staff

\begin{tabular}{llccc}
\hline & Difficulties to Nurse & & P-value \\
\hline \multirow{2}{*}{ Access site } & Central 25 pt & Yes & $25(100 \%)$ & $0.015^{* *}$ \\
\multirow{2}{*}{ Total } & Peripheral 25 pt & 0 & $21(84 \%)$ & \\
\hline
\end{tabular}

\section{Discussion}

Totally implantable venous access ports (TIVAP) provide comfort, convenience and safety in the application of chemotherapy, which when administered via peripheral vein, may result in complications, such as phlebitis, pain and even more severe consequences like skin necrosis and limb compartment syndrome due to extravasation of medication. ${ }^{5}$

These complications cause unnecessary conveniences or the patients, in addition to negatively affecting the quality of life of cancer patients. Unlike in externalized tunneled catheters (e.g., Hickman catheter), central venous arm ports show a lower infection rate, long duration of patency, and better quality of life which is important, especially in the often immunocompromised patient. ${ }^{6}$

The sites most commonly used for the insertion of these devices are currently the veins of the superior vena cava system (internal jugular and subclavian) with the reservoir positioned in the anterior chest region. These techniques are proven safe and have become even more effective after the systematic use of ultrasound guided access, with a significant reduction in cannulation failure rates, inadvertent puncture of carotid artery, hematoma formation, hemothorax or pneumothorax when compared to the technique based on the use of anatomical landmarks. ${ }^{7}$

Brachial insertion ports are safely implanted in peripheral veins, especially the basilic vein, with easy maintenance and low morbidity, since the rates of severe perioperative complications related to puncture site or pneumothorax and hemothorax are zero. Risks associated with catheter fracture between the clavicle and the first rib (pinch-off syndrome) also appear to be reduced by the use of this technique. ${ }^{4}$

Devices with the reservoir implanted in the arm offer an interesting alternative for patients with gross tumors or exposure to radiation therapy in cervical and / or anterior chest regions which contraindicate the port implantation in the conventional position. Moreover, they avoid femoral vein catheterization, 
which is greatly associated with infectious and thrombotic complications. Another possible advantage of the brachial port insertion includes better cosmetic results through avoiding scars in more exposed and visible regions. ${ }^{4}$

In our study, an excellent technical success rate of $100 \%$ was observed. Similar success rates were presented in a study with 299 patients undergoing radiological port placement via the jugular or subclavian vein, respectively undergoing radiological arm port placement in 109 patients. This study confirms already published data showing higher technical success. ${ }^{8}$

During intraoperative period the group of central approach required $\mathrm{O}_{2}$ mask and Trendelenburg positioning was mandatory. The face was covered by towels making the patient irritable while in peripheral approach this was not required, and the patient can breathe freely without covering his face.

Although incidence of accidental intraoperative complications, such as arterial puncture, pneumothorax, and hemothorax is low in other reports in the literature (pneumothorax 1.5\%$3.1 \%$; arterial puncture $3.1 \%-4.9 \%$; total $6.2 \%$ $10.7 \%),{ }^{9}$ they cannot be eliminated completely in jugular approach. In this regard, upper arm port implantation has several physical advantages. Firstly, these complications do not occur in upper arm implantation owing to anatomical reasons. Secondly, unlike subclavian, internal jugular, or femoral vein punctures, there aren't any anatomical landmarks to guide the upper arm access, forcing an operator to use ultrasound, and consequently eliminating inadvertent arterial puncture risk. In the present study, the difference between the incidences of these preprocedural complications was significant. ${ }^{10}$

The overall post-operative complication rate was the same in both groups, however, "Pinchoff syndrome" does not occur during upper arm implantation because of anatomical reasons. Distal catheter migration from the puncture point is also unlikely to occur in the straightforward upper arm lines because there are no steep turning sections causing tension from an elastic restoring force; such complications are usually observed in internal jugular procedures. ${ }^{2}$

The most frequently observed late complications in this study were vein thrombosis (6\%), infection $(8 \%)$ and catheter occlusion (6\%). Publications regarding pectoral ports report lower rates of thrombotic complications than arm ports. The reason for higher thrombotic complications may lie in the longer distance and the smaller diameter of the crossed vessels if the port is placed peripherally and this would be a potential disadvantage of this technique when compared to cervical insertion.

Kuriakose et al. reported a higher incidence of arm venous thrombosis in patients with a peripherally placed port $(11.4 \%)$ than in those with a chest port $(4.8 \%)$. The incidence of arm venous thrombosis in our series $(12 \%)$ is compatible with that of peripherally placed port. ${ }^{11}$ The only thrombotic complication was a superficial thrombophlebitis of the basilic vein, confirmed by Doppler ultrasound. After one week of full anticoagulation, patients had complete resolution of the edema, and the treatment was continued for 3 months with no further complications. The catheter remained functioning throughout the treatment period. There are currently no definite recommendations or guidelines for the use of prophylactic anticoagulation therapy to prevent thromboembolic complications. ${ }^{12}$

Catheter infection and occlusion rates were also observed, and they were mostly attributed to inadvertent mishandling of the port during the service interval; and this led us to recommend more effort to teach and train medical staff on how to use the devices safely and properly, with emphasis on how to access the port under strictly sterile conditions and on filling the system with heparinized sodium chloride after usage to prevent potential problems in the long-term management of the port. ${ }^{13}$

No complications regarding a breakdown of the port system were reported, and there was minimal drug leak (one case only) noted over the observation period of 1 to 24 months in our series. This is probably because the main healthcare providers privileged to deal with TIVP patients at our institute are all highly trained in the use of several types of TIVP systems, and thereby mechanical damage to the port system could be avoided.

We found no significant differences for some questions addressed in the questionnaire compared to the findings of Goltz et al. ${ }^{14} 76 \%$ of the patients from the peripheral approach preferred this site compared to $12 \%$ with the central approach. Moreover, $28 \%$ of the patients (mainly females) in the central approach group said that it would be better if they had had their port peripherally compared to $(2 \%)$ in the peripheral approach who wanted it on chest. They reasoned that upper arm implantation does not leave scars on the neck or chest, which may prevent patients from wearing wide-open neck clothes because of cosmetic concerns, in addition to more dignified exposure during the injection session that usually occurs in day case wards.

Finally, in our study the patients complained less about any unpleasant sensation associated with 
the port, and reported lower impact caused by the device on daily activities, such as moving the arm and wearing clothes. The level of satisfaction with the aesthetic results observed among patients in our study was higher in peripherally placed ports than in centrally located ones $(p=0.0001)$.

\section{Conclusion}

In conclusion, for patients with peripheral veins no longer suitable to accept an indwelling needle, TIVP is safe and suitable for long-term use for chemotherapy. The brachial port implantation is a feasible option with low surgical risk, low intraoperative complications, similar rates of postoperative and late complications compared to existing data in the central access approach, and can provide more safety and comfort benefits to both medical professionals and cancer patients.

Our results showed a high level of patient satisfaction and quality of life with the brachial catheter insertion, and almost all the patients enrolled would recommend this approach to others.

Our study demonstrated only an initial evaluation of a technique not often used in our practice, but can be employed safely and presents satisfactory results.

We hope that this procedure will become more common and eventually be validated in prospective multicenter randomized clinical trials regarding its non $\neg$ inferiority or superiority to the internal jugular procedures with respect to safety, maintenance of quality of life, and cost-effectiveness.

\section{References}

1. Nocito A, Wildi S, Rufibach K, Clavien PA, Weber $M$ : Randomized clinical trial comparing venous cutdown with the Seldinger technique for placement of implantable venous access ports. British Journal of Surgery. 2009; 96(10): 112934.

2. Shiono M, Takahashi S, Kakudo Y, Takahashi M, Shimodaira H, Kato S, et al: Upper arm central venous port implantation: A 6-year single institutional retrospective analysis and pictorial essay of procedures for insertion. PLOS ONE. 2014; 9(3): e91335.

3. Smok F, Macholz M, Gorisch G, Grunewald T, Taeger CD, Friedrich $O$, et al: Surveillance of nosocomial Clostridium difficile infection (nCDI) in a large tertiary community hospital. International Journal of Infectious Diseases. 2016; 45: 279.

4. Fonseca IYI, Krutman M, Nishinari K, Yazbek
G, Teivelis MP, Bomfim GAZ, et al: Brachial insertion of fully implantable venous catheters for chemotherapy: Complications and quality of life assessment in 35 patients. Einstein (Sao Paulo). 2016; 14(4): 473-9.

5. Kumar S: The Association of Health Literacy with the Management of Type 2 Diabetes: University of North Texas Health Science Center at Fort Worth. 2017.

6. Nakamura T, Sasaki J, Asari Y, Sato T, Torii S, Watanabe M: Complications after implantation of subcutaneous central venous ports (PowerPort $(\circledR))$. Annals of medicine and Surgery (2012). 2017; 17: 1-6.

7. DePietro DM, Trerotola SO: Central venous access. IR Playbook: Springer. 2017: 115-30.

8. Goltz JP, Scholl A, Ritter CO, Wittenberg G, Hahn D, Kickuth R: Peripherally placed totally implantable venous-access port systems of the forearm: Clinical experience in 763 consecutive patients. Cardiovascular and Interventional Radiology. 2010; 33(6): 1159-67.

9. McGee DC, Gould MK: Preventing complications of central venous catheterization. New England Journal of Medicine. 2003; 348(12): 1123-33.

10. Ezaru CS, Mangione MP, Oravitz TM, Ibinson JW, Bjerke RJ: Eliminating arterial injury during central venous catheterization using manometry. Anesthesia \& Analgesia. 2009; 109(1): 130-4.

11. Kuriakose P, Colon-Otero G, Paz-Fumagalli R: Risk of deep venous thrombosis associated with chest versus arm central venous subcutaneous port catheters: A 5-year single-institution retrospective study. Journal of Vascular and Interventional Radiology. 2002; 13(2): 179-84.

12. Shaw C: Central line management and intestinal failure. Contemporary Small Bowel Transplantation. 2018: 1-27.

13. Matey L, Camp-Sorrell D: Venous access devices: Clinical rounds. Asia-Pacific Journal of Oncology Nursing. 2016; 3(4): 357-64.

14. Fonseca IY, Krutman M, Nishinari K, Yazbek G, Teivelis MP, Bomfim GA, et al: Brachial insertion of fully implantable venous catheters for chemotherapy: Complications and quality of life assessment in 35 patients. Einstein (Sao Paulo). 2016; 14(4): 473-9. 